\title{
Recurrent Pseudotumoral Demyelinating Disease in an Adolescent Patient
}

Osman Farooq ${ }^{1^{*}}$, David Hojnacki ${ }^{2}$, Lucia Balos ${ }^{3}$ and Bianca Weinstock-Guttman ${ }^{2}$

${ }^{1}$ Women \& Children Hospital of Buffalo, SUNY University of Buffalo, USA

2Jacobs MS Center, SUNY University at Buffalo, USA

${ }^{3}$ Department of Pathology, SUNY University at Buffalo, USA

"Corresponding author: Osman Farooq MD, Women \& Children Hospital of Buffalo, Department of Neurology, 219 Bryant Street, Buffalo, NY 14222, USA, Tel: 716-878-7840; E-mail: ofarooq3@buffalo.edu

Received date: Jun 03, 2014, Accepted date: Jul 09, 2014, Published date: Jul 11, 2014

Copyright: (C) 2014 Farooq O, et al. This is an open-access article distributed under the terms of the Creative Commons Attribution License, which permits unrestricted use, distribution, and reproduction in any medium, provided the original author and source are credited.

\section{Introduction}

Multiple sclerosis (MS) is a chronic progressive inflammatory demyelinating and degenerative condition affecting the nervous system. Typical magnetic resonance imaging (MRI) findings consist of multiple asymmetric white matter lesions, with a predilection for periventricular and subcortical white matter [1]. The number and extent of lesion accumulation can be predictive of the disease course. As opposed to these multiple white matter lesions seen in MS, in the pseudotumoral inflammatory demyelinating form the onset of disease is characterized by a large isolated mass, typically in the white matter, usually in the brain hemispheres [2]. When these lesions are over 20 $\mathrm{mm}$ in diameter, they are defined as tumefactive, or pseudotumoral demyelinating lesions [1]. This form of demyelinating disease is rare, and even more so in the pediatric population. Here we present a case of an adolescent boy with pseudotumoral MS with recurrence several years later, while on no disease modifying therapy with multifocal pseudotumoral lesions. To our knowledge this is the first pediatric case of recurrent pseudotumoral disease occurring within several years.

\section{Case Report}

A previously healthy 12 year old boy presented with a persistent and worsening right frontal headache. The parents report a longstanding history of focal headaches, localized to the right frontal region, however in recent days these headaches had increased in frequency and severity. There was no reported photophobia, phonophobia, or visual disturbances reported. The general physical as well as neurological exams were within normal limits.

The MRI of the brain (Figures 1a and $1 \mathrm{~b}$ ) revealed a large mass with a central, well circumscribed cystic area. A ring-like enhancement pattern was seen as well as a mass effect. Spinal imaging did not reveal abnormalities or evidence of similar lesions.

Surgery was attempted to decompress the suspected tumor. However during the surgical procedure, no demarcation could be made between normal and abnormal tissue, therefore a biopsy of the area in question was performed. Fluid from the cystic decompression failed to reveal any evidence of tumor/malignancy. Cytology was negative. Pathological evaluation of the biopsy specimen revealed a markedly hypercellular with somewhat congested vasculature tissue. A perivascular lymphocyte component could not readily be identified. However, the CD68 immunohistochemistry for macrophages diffusely stained the majority of the cells in the lesion and identified clusters of macrophages in the Virchow-Robin spaces as well (Figures $2 a$ and $2 b$ ). The differential diagnosis of granular cell astrocytoma and demyelinating process was considered and preference to demyelination given due the major component of the lesion comprising macrophages.

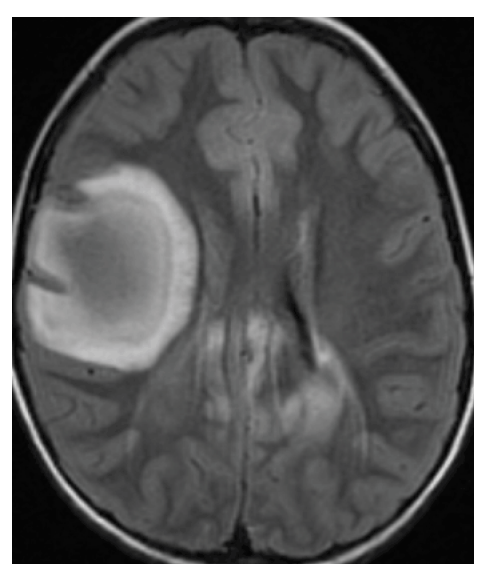

Figure 1a: Axial FLAIR MRI, revealing a large hyperintense lesion in the right periventricular region extending to the internal capsule.

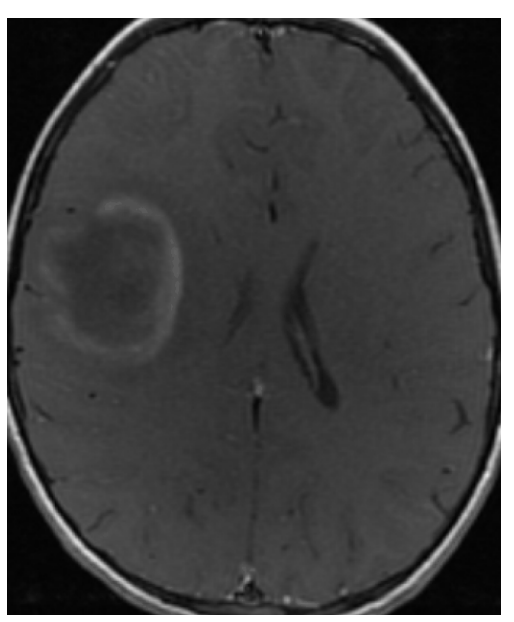

Figure 1b: Axial T1 post-contrast, revealing with patchy contrast enhancement in an incomplete/open ring-enhancement pattern. 
Citation: Farooq O, Hojnacki D, Balos L, Weinstock-Guttman B (2014) Recurrent Pseudotumoral Demyelinating Disease in an Adolescent Patient. J Mult Scler 1: 111. doi:10.4172/jmso.1000111

Page 2 of 4

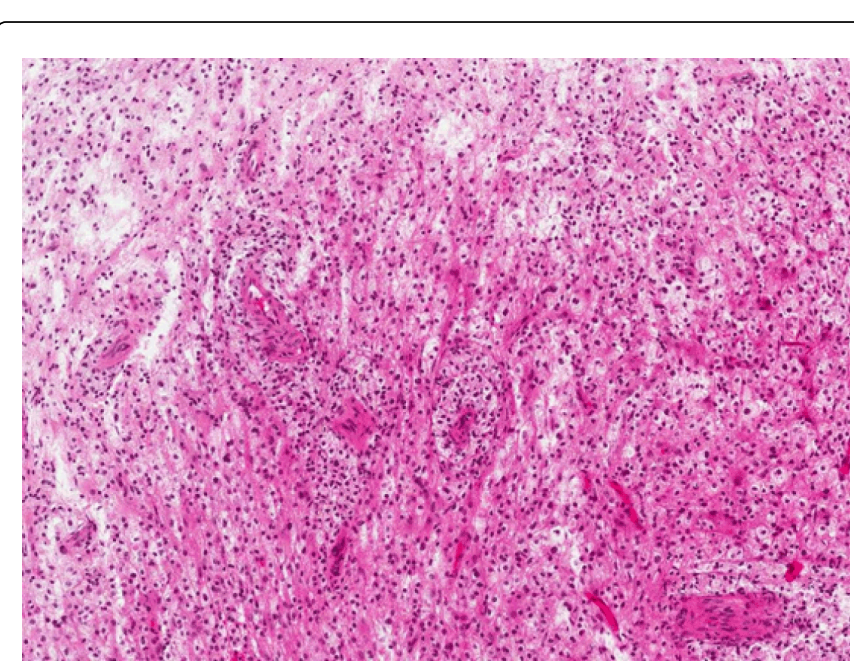

Figure 2a: H\&E stained section of biopsy showing the marked hypercellularity of the tissue comprising clear and granular cells. Granular cells surround blood vessels in the Virchow-Robin space.

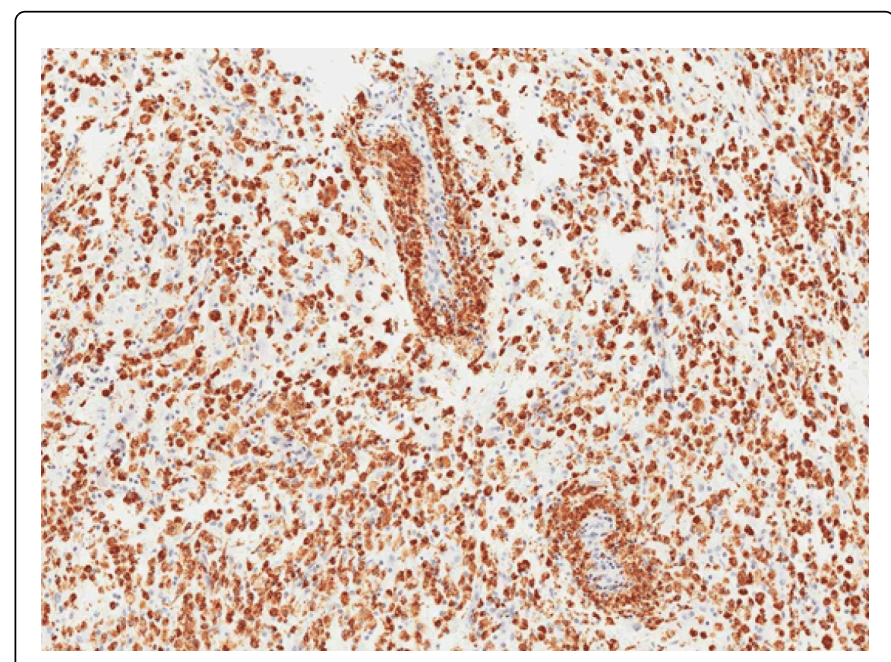

Figure 2b: CD68 immunohistochemisty demonstrates the diffuse infiltration of the tissue by macrophages. The Virchow-Robin space vascular cuffing is also pronounced.

A visual evoke response (VER) revealed a delayed P100 on the left. As this lesion was seen in isolation with histological evidence of inflammation and demyelination, he was diagnosed as having a pseudotumoral demyelinating process. He was treated with steroids, and the lesion decreased significantly in size. Clinically he returned to his baseline. Repeat MRIs and VERs were performed on a yearly basis and were stable.

Five years later, he experienced a sudden worsening of symptoms, at which time he experienced diffuse right sided hemiplegia as well as a right temporal visual field defect. This occurred three weeks after treatment with amoxicillin for a pharyngeal infection. An MRI (Figure 3a-3f) revealed multiple large white matter lesions with surrounding edema in the left frontal and temporal regions as well as in the right fronto-temporal region. There was evidence of contrast enhancement. Cerebrospinal fluid (CSF) analysis was normal, without oligoclonal bands or NMO IgG antibodies (serum and CSF). His symptoms showed only modest improvement with steroids. He was then treated with plasma exchange followed by intravenous immunoglobulin (IVIG), which aided in the speed of recovery. Because the recurrence and severity of the second demyelinating event, the patient was initiated on monthly IVIG $0.4 \mathrm{gr} / \mathrm{kg}$ for 6 months with the consideration of close monitoring. Repeat MRI's improved significantly with no new lesions within a follow up of almost 1 year.

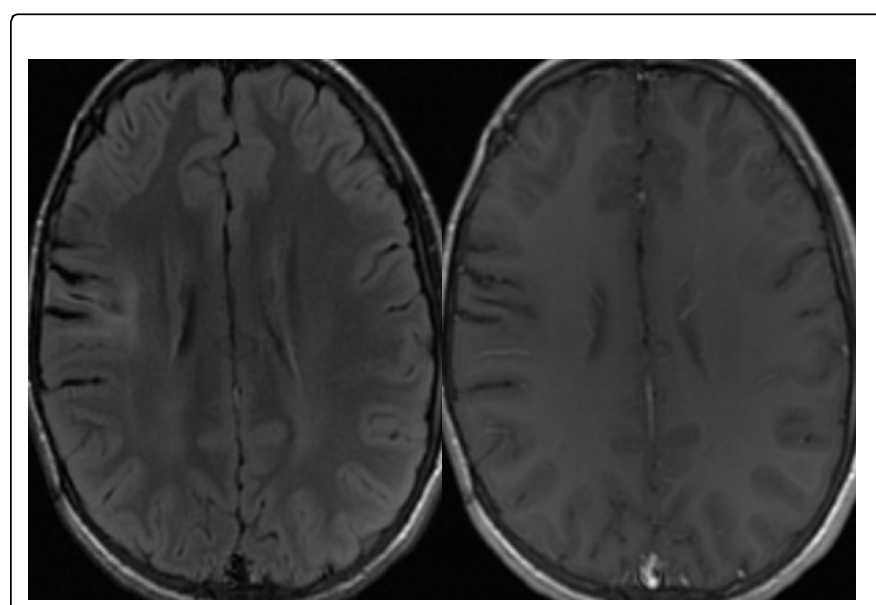

Figure 3a and 3b: Axial FLAIR (3a) and corresponding T1 postcontrast ( $3 \mathrm{~b})$ revealing encephalomalacia in the area of the previous lesion in right periventricular region.

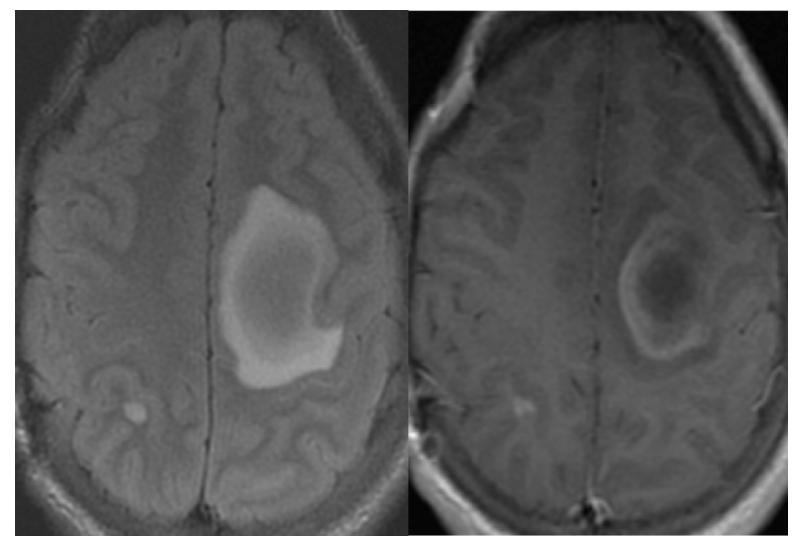

Figure 3c and 3d: Axial FLAIR (3a) revealing a large lesion in the left frontal region with surrounding edema as well as a small lesion in the right parietal region. The corresponding T1 post-contrast (3b) revealing an incomplete/open ring-enhancement pattern in the left frontal lobe as well as enhancement in the small lesion in the right parietal region. 


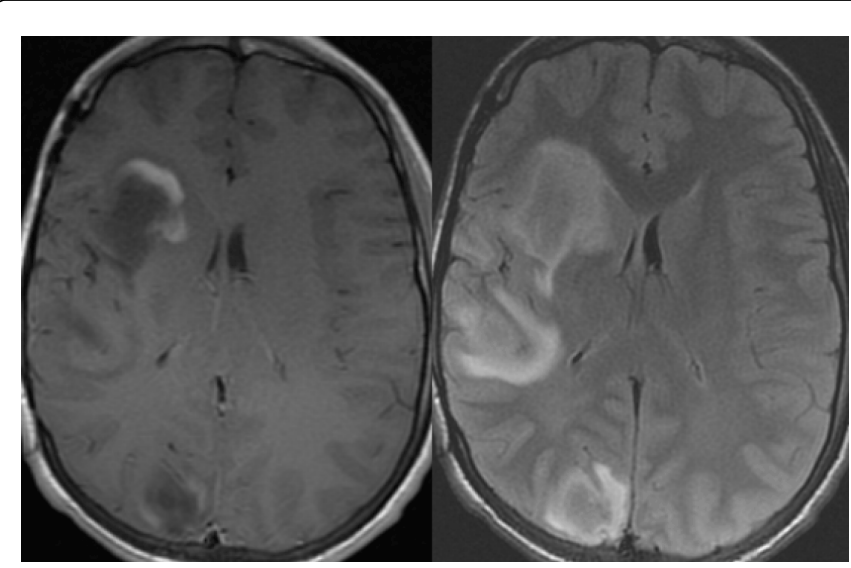

Figure 3e and 3f: Axial FLAIR (3a) revealing two large lesions in the right temporal as well as occipital regions with surrounding edema. The corresponding $\mathrm{T} 1$ post-contrast ( $3 \mathrm{~b}$ ) revealing incomplete/open ring-enhancement pattern in both lesions.

\section{Discussion}

Tumefactive demyelinating lesions are thought to represent either a variant of MS or a unique form of isolated demyelinating disease [3,4]. Some patients are found to have developmentally immature myelin basic protein, pointing toward the possibility of a genetic predisposition to develop more severe demyelination [5]. A vaccination or antecedent infection may precede the demyelinating event as often seen in acute disseminated encephalomyelitis (ADEM), suggesting an autoimmune underlying mechanism [4].

Tumefactive demyelinating lesions can often be misdiagnosed as tumors or abscesses. Ravi et al. [6] described a case of a 10 year old girl who had a surgical resection of a suspected glioblasoma multiforme, and was scheduled to receive chemotherapy when histological evaluation revealed evidence of demyelination. Neuroimaging may help narrow the differential diagnosis however a biopsy is often required for a definite diagnosis. Tumefactive demyelinating lesions are seen as well-demarcated, hypodense areas on computed tomography (CT) and are hyperintense on T2- and relatively hypointense on T1weighted MRI. Ring enhancement with gadolinium is a characteristic feature of tumefactive demyelinating lesions $[7,8]$. The most frequent pattern seen upon gadolinium enhancement is an open/incomplete ring, where the open portion abuts the gray matter of the cortex or basal ganglia [1]. Another feature that can help differentiate plaques from tumors are the presence of dilated vascular structures in the middle of the lesion, which are thought to be enlarged veins draining into swollen subependymal veins [6,9]. Although non-specific, factors such as the size and location of the lesions, the relative lack of mass effect, and the edema are other helpful radiological findings [10,11]. Magnetic resonance spectroscopy (MRS) can help narrow the differential diagnosis, however it does not effectively differentiate tumefactive demyelinating lesions from neoplasms $[12,13]$.

Presenting symptoms generally depend on the lesion's size and location. Typical features include an acute or subacute onset, quick progression of neurological deficits with frequent involvement of cognitive functions. In addition, deficits of symbolic functions (apraxia, agnosia, aphasia) can occur when the frontal regions are involved and acute memory dysfunction in case of involvement of the temporal regions. Hemiparesis, of variable severity, and of sensorial and visual disorders, are also frequent. Another typical feature is the appearance of seizures when there is a cortical involvement $[2,14]$. Headaches of varying degrees can also be a common presenting symptoms, as in our patient.

The differential diagnosis can be quite problematic, particularly in the initial stages of the disease. The main entities to keep in mind would be tumor and ADEM, and to a lesser extent progressive multifocal leukoencephalopathy (PML), abscess or parasitic cysts. The tumor, which can be easily mistaken for a demyelinating lesion, is lymphoma. The brain lymphoma is usually unifocal, but in some cases can be multicentric. ADEM usually appears with small and bilateral lesions, which are generally symmetric, but in rare cases large isolated lesions can be observed [4]. Abscesses and parasitic cysts can have aspects similar to inflammatory pseudotumoral lesions, but the characteristics of enhancement are different as the ring around the cystic cavity is usually complete, whereas in the demyelinating lesion, it is mostly "incomplete" [2].

The long-term evolution of these inflammatory lesions is not well defined. It is estimated that about one third of the patients have a monophasic course with complete recovery, one third have a monophasic course with prolonged evolution and residual deficits, and the remaining third evolves to MS [1].

The relative paucity of reported cases of tumefactive demyelination in children likely represents an underestimate of the actual frequency of cases. In 2002, McAdam et al., [15] reviewed 12 cases in the literature, plus 4 cases observed personally. Morin et al., [16] described 3 additional cases. Puri and associates [17] described a case of recurrent pseudotumoral MS, with two episodes occurring 6 months apart. Our case is unique due to the prolonged interval in between recurrence (5 years). In addition, upon recurrence the symptomatology and clinical severity was considerably worse, requiring plasma exchange as steroids alone were not sufficient. Typically with pseudotumoral MS solitary lesions are found, whereas in our patient there were multiple lesions at recurrence. This raised the concern for the need of a preventative medication, however following six monthly IVIG cycles the patient has shown no evidence of clinical or radiological recurrence of disease for 1 year.

\section{Summary}

Pseudotumoral MS can be a diagnostic challenge. The diagnosis in itself can be rare, however our case is unique because there was clinical recurrence after 5 years while on no therapy and because of the early age of presentation. Moreover, upon recurrence our patient exhibited multiple lesions with pseudotumoral features, which is unusual for pseudotumoral MS. Initiation of a preventive disease modifying therapy (DMT) was deferred because the atypical presentation and the 5 year initial remission.

\section{References}

1. Altintas A, Petek B, Isik N, Terzi M, Bolukbasi F, et al. (2012) Clinical and radiological characteristics of tumefactive demyelinating lesions: follow-up study. Mult Scler 18: 1448-1453.

2. Comi G (2004) Multiple sclerosis: pseudotumoral forms. Neurol Sci 25 Suppl 4: S374-379. 
Citation: Farooq O, Hojnacki D, Balos L, Weinstock-Guttman B (2014) Recurrent Pseudotumoral Demyelinating Disease in an Adolescent Patient. J Mult Scler 1: 111. doi:10.4172/jmso.1000111

Page 4 of 4

3. Poser S, Lüer W, Bruhn H, Frahm J, Brück Y, et al. (1992) Acute demyelinating disease. Classification and non-invasive diagnosis. Acta Neurol Scand 86: 579-585.

4. Kepes JJ (1993) Large focal tumor-like demyelinating lesions of the brain Intermediate entity between multiple sclerosis and acute disseminated encephalomyelitis? A study of 31 patients. Ann Neurol 33: 18-27.

5. Wood DD, Bilbao JM, O'Connors P, Moscarello MA (1996) Acute multiple sclerosis (Marburg type) is associated with developmentally immature myelin basic protein. Ann Neurol 40: 18-24.

6. Riva D, Chiapparini L, Pollo B, Balestrini MR, Massimino M, et al. (2008) A case of pediatric tumefactive demyelinating lesion misdiagnosed and treated as glioblastoma. J Child Neurol 23: 944-947.

7. Ernst T, Chang L, Walot I, Huff K (1998) Physiologic MRI of a tumefactive multiple sclerosis lesion. Neurology 51: 1486-1488.

8. Masdeu JC, Quinto C, Olivera C, Tenner M, Leslie D, et al. (2000) Openring imaging sign: highly specific for atypical brain demyelination. Neurology 54: 1427-1433.

9. Cha S, Pierce S, Knopp EA, Johnson G, Yang C, et al. (2001) Dynamic contrast-enhanced $\mathrm{T} 2{ }^{*}$-weighted $\mathrm{MR}$ imaging of tumefactive demyelinating lesions. AJNR Am J Neuroradiol 22: 1109-1116.

10. Dagher AP, Smirniotopoulos J (1996) Tumefactive demyelinating lesions. Neuroradiology 38: 560-565.
11. Kiriyama T, Kataoka H, Taoka T, Tonomura Y, Terashima M, et al. (2011) Characteristic neuroimaging in patients with tumefactive demyelinating lesions exceeding $30 \mathrm{~mm}$. J Neuroimaging 21: e69-77.

12. Xia L, Lin S, Wang ZC, Li SW, Xu L, et al. (2009) Tumefactive demyelinating lesions: nine cases and a review of the literature. Neurosurg Rev 32: 171-179.

13. Malhotra HS, Jain KK, Agarwal A, Singh MK, Yadav SK, et al. (2009) Characterization of tumefactive demyelinating lesions using MR imaging and in-vivo proton MR spectroscopy. Mult Scler 15: 193-203.

14. Lucchinetti CF, Gavrilova RH, Metz I, Parisi JE, Scheithauer BW, et al. (2008) Clinical and radiographic spectrum of pathologically confirmed tumefactive multiple sclerosis. Brain 131: 1759-1775.

15. McAdam LC, Blaser SI, Banwell BL (2002) Pediatric tumefactive demyelination: case series and review of the literature. Pediatr Neurol 26: 18-25.

16. Morin MP, Patenaude Y, Sinsky AB, Banwell B, Sébire G (2011) Solitary tumefactive demyelinating lesions in children. J Child Neurol 26: 995-999.

17. Puri V, Chaudhry N, Gulati P, Tatke M, Singh D (2005) Recurrent tumefactive demyelination in a child. J Clin Neurosci 12: 495-500. 